\title{
Kernos
}

Revue internationale et pluridisciplinaire de religion grecque antique

$22 \mid 2009$

Varia

\section{Alberto Bernabé, Ana Isabel Jiménez San Cristóbal, Instructions for the Netherworld. The Orphic Gold Tablets}

\section{Vinciane Pirenne-Delforge}

\section{(2) OpenEdition \\ 1 Journals}

\section{Édition électronique}

URL : http://journals.openedition.org/kernos/1811

DOI : 10.4000/kernos. 1811

ISSN : 2034-7871

\section{Éditeur}

Centre international d'étude de la religion grecque antique

\section{Édition imprimée}

Date de publication : 1 janvier 2009

Pagination : 334-336

ISSN : 0776-3824

\section{Référence électronique}

Vinciane Pirenne-Delforge, «Alberto Bernabé, Ana Isabel Jiménez San Cristóbal, Instructions for the Netherworld. The Orphic Gold Tablets », Kernos [En ligne], 22 | 2009, mis en ligne le 15 septembre 2011, consulté le 21 septembre 2020. URL : http://journals.openedition.org/kernos/1811 ; DOI : https://doi.org/10.4000/kernos. 1811

\section{Ce document a été généré automatiquement le 21 septembre 2020}




\title{
Alberto Bernabé, Ana Isabel Jiménez San Cristóbal, Instructions for the Netherworld. The Orphic Gold Tablets
}

\author{
Vinciane Pirenne-Delforge
}

\section{RÉFÉRENCE}

Alberto BERNABÉ - Ana Isabel JIMÉNEZ SAN CRISTÓBAL, Instructions for the Netherworld. The Orphic Gold Tablets, Leiden, Brill, 2008. 1 vol. 16,5 厲 $24,5 \mathrm{~cm}, 379$ p. (Religions in the Graeco-Roman World, 162). ISBN : 978-90-04-16371-3.

La version espagnole de ce livre date de 2001. En 2004, A. Bernabé a également publié l'importante édition des Poetae Epici Graeci. Testimonia et Fragmenta II. Orphicorum et Orphicis similium testimonia et fragmenta fasc. 2 (Bibliotheca Teubneriana) et l'édition des textes que reprend l'Appendice 1 de l'ouvrage ici recensé tient évidemment compte de ce travail paru entre-temps. Il s'agit donc d'une analyse des tablettes "orphiques ", parfaitement maîtrisée sur le plan philologique, par l'un des meilleurs connaisseurs de la littérature "orphique » au sens le plus large du terme, et de l'une de ses proches collaboratrices. L'usage des guillemets autour du qualificatif « orphique » - qui est mien - pose évidemment d'emblée le problème central de l'ouvrage, à savoir l'ancrage des tablettes en or, mises au jour dans des tombes, dans le cadre d'un courant de type sectaire sous la houlette d'Orphée. Pour les A., la cause est entendue et c'est «without hesitation" (p. 2) qu'ils identifient les utilisateurs de ces documents au groupe minoritaire des sectateurs du mouvement religieux orphique. Il est vrai que ce point de vue est aujourd'hui largement partagé, même si des nuances émergent dans le petit monde des exégètes de ces tablettes, et que ses adversaires les plus radicaux ne sont plus très nombreux ${ }^{1}$. Il n'en reste pas moins que l'absence totale d'Orphée de ces textes continue de poser question. Et quand bien même balaierait-on cette absence d'un 
revers de main, la prétendue "unité de croyance» des utilisateurs des tablettes reste difficile à établir. C'est pourtant une telle unité que les A. décèlent derrière tous ces documents. Mais, quelle que soit la qualité de l'érudition mise en œuvre - et elles est grande, - leur vision unitaire d'une « religion orphique » (« the adepts of this religion », p. 7) contraste avec le fonctionnement même du système religieux des Grecs, étranger à ce type de référent quasi dogmatique. D'aucuns y verront la preuve du caractère sectaire de la démarche - par un raisonnement qui frôle la circularité, - d'autres y ancreront leur réticence à situer parmi les représentations religieuses des Grecs ce type de référent. En fait, la seule certitude qui ressort de tous ces documents est davantage rituelle : derrière la plupart d'entre eux se profile un rituel de type initiatique auquel s'est soumis le défunt durant sa vie et qui lui permet d'accéder à un statut bienheureux dans l'au-delà. L'unité apparente qui sous-tend bon nombre de tablettes émane assurément de la nature initiatique de ce changement de statut, lié à un enseignement à garder en mémoire. Cela implique-t-il pour autant que ce soit le même contexte initiatique qui ait joué à chaque fois?

2 Quoi qu'il en soit de ce débat dont on n'est sans doute pas près de sortir sans de nouveaux documents, l'ouvrage rendra d'éminents services à tous ceux qu'intéressent ces questions. Il est construit en douze chapitres, qui forment un ample commentaire des documents édités en annexe. Cela signifie que le texte grec doit toujours être recherché dans cette annexe et que seules des traductions sont produites au fil du commentaire. Quant au contenu de ce commentaire, les neuf premiers chapitres sont conçus en fonction de la typologie thématique des tablettes élaborée par les A., les textes correspondant à cette typologie étant donc systématiquement convoqués dans les chapitres adéquats. Ainsi, le $1^{\mathrm{er}}$ chapitre se concentre sur l'arrivée dans l'au-delà, qui caractérise bon nombre de documents. Le $2^{\mathrm{e}}$ chapitre aborde le rituel auquel le défunt se soumet pour accéder à son heureuse destinée, qui apparaît dans les deux tablettes de Pelinna. Le $3^{\mathrm{e}}$ chapitre se fonde sur une des tablettes de Thourioi où le défunt voit décrit son parcours post mortem dont l'objectif semble considéré comme atteint. Le $4^{\mathrm{e}}$ chapitre reprend d'autres tablettes de Thourioi où intervient Perséphone dans l'accueil du mort. Le $5^{\mathrm{e}}$ chapitre étudie la tablette la plus récente du corpus, trouvée à Rome, et qui est comme une variation sur le thème du chapitre précédent. Le $6^{e}$ chapitre repart à Thourioi et se penche sur la plus énigmatique des tablettes qui y ont été trouvées, portant une accumulation de termes signifiants entourés d'éléments incompréhensibles. Il s'agirait de la dissimulation sciemment orchestrée d'éléments mystiques à la seule intention des initiés. Le $7^{\mathrm{e}}$ chapitre analyse deux textes de Pherai et aborde la question des «mots de passe » énoncé par le défunts pour accéder à l'audelà. L'une des deux tablettes a été tout récemment publié par R. Parker et M. Stamatopoulou ${ }^{2}$ et fait surgir une Déméter Chthonia et une Mètèr Oreia dont les initiations sont invoquées par le mort en vue de rejoindre le thiase des mystes. Le «Bacchos » qui viendrait placer ce document dans la série " orphico-bacchique » ou " orphico-dionysiaque » est une restitution. Le texte le plus récemment mis au jour est donc venu compliquer encore le tableau, sans permettre de trancher définitivement le débat évoqué ci-dessus. Le $8^{\mathrm{e}}$ chapitre examine diverses tablettes provenant de Crète et d'ailleurs, dont le point commun est la brièveté, un salut aux dieux des enfers et la référence aux mystes.

Les quatre derniers chapitres sont indépendants de la typologie proprement dite. Le $9^{\mathrm{e}}$ chapitre résume ce que ces tablettes permettent de connaître de la destinée de l'âme, 
ce qui n'est évidemment possible qu'en postulant une même représentation d'arrièreplan pour l'ensemble, une option confirmée par le $10^{\mathrm{e}}$ chapitre qui réaffirme la nature orphique de tous ces documents. Le $11^{\mathrm{e}}$ chapitre collecte des parallèles dans d'autres cultures, comme le Livre des morts égyptien, le «Grand voyage de l'âme " hittite, certains aspects de l'eschatologie védique ou iranienne, et quelques tablettes qui viennent de Gaule et du monde punique. Le $12^{\mathrm{e}}$ et dernier chapitre aborde les questions littéraires liées aux tablettes, ce qui ouvre la réflexion sur le problème d'un éventuel modèle et de la relation de ces textes à un rituel antérieur et à leur propre utilisation rituelle. Le $1^{\mathrm{er}}$ appendice livre l'édition des tablettes et le $2^{\mathrm{e}}$, un ensemble de dessins reprenant des documents iconographiques censés renvoyer, peu ou prou, à l'orphisme ( «approximations to the religious world of the Orphic tablets from the viewpoint of iconography », p. 275), un ensemble disparate qui fera certainement grincer quelques dents parmi les spécialistes des images.

La structure même de l'ouvrage est donc totalement tributaire de la conviction qu'une «religion orphique» unifiée a existé entre la période classique - au moins - et la période romaine, dans l'ensemble du monde grec. Cette religion avait ses fidèles qui, en marge du système religieux des cités, croyaient en la métempsycose et en la possibilité d'un au-delà bienheureux. Toutefois, les tablettes d'or ne permettent pas d'identifier la croyance en la métempsycose (la « libération » qu'elles invoquent n'est pas forcément un sortie du cycle des incarnations) et l'initiation éleusinienne promettait elle aussi un au-delà bienheureux. Faut-il donc aussi catégoriquement dissocier ce vaste ensemble documentaire du polythéisme grec traditionnel, dont bon nombre de réflexions des A. montrent qu'ils ont une vision assez datée ? Pour ma part, je me réjouis de disposer de cette remarquable somme d'information, mais je ne suis pas certaine que le dernier mot soit dit sur ces questions complexes.

\section{NOTES}

1. Voir sur ce point le compte rendu critique que Claude Calame a donné de l'ouvrage récent de Fr. Graf et S.I. Johnston, et de celui de M. Tortorelli-Ghidini, dans la livraison précédente de Kernos : 21 (2008), p. 299-311.

2. R. PARKer \& M. STAMATOPOUlou, «A new funerary gold leaf from Pherai », AEphem (2004) [2007], p. 1-32. 


\section{AUTEURS}

VINCIANE PIRENNE-DELFORGE

F.R.S.-FNRS - Université de Liège 